\title{
Can we distinguish quark stars from neutron stars with measurements of global properties?
}

\author{
Ang $\mathrm{Li}^{1, *}$ \\ ${ }^{1}$ Department of Astronomy, Xiamen University, Xiamen, Fujian 361005, China
}

\begin{abstract}
The phase state of the dense stellar matter is an exciting topic in the area of nuclear astrophysics. It may be probed by observed properties of neutron stars from, for example, the currently operating satellites (NICER, Neutron star Interior Composition Explorer) and the gravitational-wave laser interferometers (Advanced LIGO, Virgo, and KAGRA). Based on our recent constrained parameter spaces of the equation of states of neutron stars and quark stars from LIGO/Virgo and NICER, we discuss the important role of an even-accurate determination of the stellar radius for distinguishing possible quark stars from neutron stars and our understanding of the QCD phase transition at finite density.
\end{abstract}

\section{Introduction}

Neutron stars are the remnants of the gravitational collapse of very massive stars during the supernova event. They may have masses in the range of $M \sim 1-3 M_{\odot}$ and radii of $R \sim$ $10-20 \mathrm{~km}$. So the matter inside the star is quite dense, with an average density exceeding the ordinary nuclear density. They are the densest and smallest stars observed in the Universe, composed of mainly nuclear matter plus a small fraction of electrons and muons. A neutron star is bound by gravity, and it is kept in hydrostatic equilibrium only by the pressure produced by the compressed nuclear matter. The hadronic matter might undergo a phase transition to quark matter inside the high-density cores of neutron stars, forming hybrid stars. In a hypothetic scenario [1, 2], neutron stars might even be self-bound with deconfined quarks constituting the entire star, i.e., quark stars. The equation of state (EOS), mainly pressure as a function of the (energy) density $p(\varepsilon)$ is informative of the composition and the inner structure of a neutron star; And experimental nuclear physics provides critical data needed to benchmark theories of dense matter EOS residing in neutron stars [3].

However, such extreme conditions make it impossible to attain the dense matter EOS by experimental methods only; several recent observational developments provide powerful constraints on the EOS and the internal composition. Among these are the measurable global properties of neutron stars, including the mass (PSR J0348+0432 [4], PSR J2215+5135 [5], PSR J0740+6620 [6]), the radius (PSR J0030+0451 [7, 8], PSR J0740+6620 [9, 10]), and the tidal deformability (GW170817) [11]. Fig. 1 demonstrates the effective constraining on the mass-radius relations from these data (all results are shown to the $90 \%$ confidence level if otherwise specified). In particular, the not-too-high tidal deformability of GW170817 can

\footnotetext{
*e-mail: liang@xmu.edu.cn
} 
exclude very stiff EOSs, the relatively large radii of PSR J0030+0451 (with a mass around the typical mass $1.4 M_{\odot}$ ) disfavors very soft EOSs. The simultaneous measurement of the masses and radii of 2-solar-mass pulsars (like the presently available PSR J0740+6620) can exclude the mass-radius parameter space from both the stiff and the soft sides, in this sense serving as an ideal probe for the EOS study of dense matter.
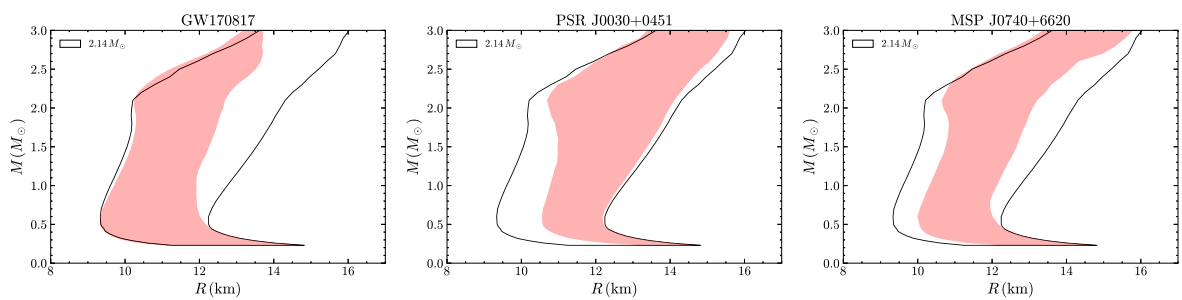

Figure 1. Posterior probability distributions (pink regions) of the mass-radius relations from the tidal deformability of GW170817 (left) and the simultaneous measure of the neutron star mass and radius of low-mass PSR J0030+0451 (middle) and high-mass PSR J0740+6620 (right), compared to the black area in each panel, obtained based on the EOSs consistent with the lower limit on the maximum mass $M_{\mathrm{TOV}}$ from the mass distribution measured for PSR J0740+6620.

The discussions in the present contribution follow the limited references of our recent works [12-18], and we mainly focus on one of the critical problems in the EOS study: Is it possible to distinguish gravity-bound (quark hybrid) neutron stars from self-bound quark stars with measurements of global properties? Since the strange quark matter in hybrid stars and quark stars are described under different stability conditions, and both of them can't coexist stably [19], a confirmation (or exclusion) of either of them can shed light on the remaining-unsolved QCD at finite baryon density [30]. We mention here that [12] made use of several nuclear many-body frameworks for various modeling of dense matter and found that the "effective" stiffness of the EOS, including the sound velocity $c_{s}=\sqrt{d P / d \varepsilon}$ and the adiabatic index $\Gamma=(\varepsilon+P)(d P / d \varepsilon) / P$, can not effectively signify the matter's composition in intermediate (nonperperturbative) densities relevant to compact stars.

\section{Models and Analysis}

Model calculations showed that massive stars (like heaviest PSR J0740+6620) could be hybrid stars with a stiff quark-matter core [20]. The parameter space for neutron star EOSs could be extended significantly through the introduction of a hadron-quark phase transition [14], while maintaining consistency with the observational data from tidal deformability of the GW170817 and GW190425 binary neutron star merger as detected by LIGO/Virgo and the mass and radius of PSR J0030+0451 and PSR J0740+6620 as detected by NICER. The EOSs for hybrid stars $[16,17]$ constrained within the Bayesian statistical approach are shown in the left panel Fig. 2. For the analysis, we have assumed that the hadron-quark phase transition is of first order, where the low-density hadronic matter described in a unified manner by the QMF EOS [21] transforms into a high-density phase of quark matter modeled by the generic "Constant-sound-speed" (CSS) parameterization [22]. The CSS parameterization is a general parametrization suitable for expressing experimental constraints in a model-independent way. The value of the sound speed is varied from $1 / \sqrt{3}$ (the perturbative QCD value) to 1 (the casual limit). The parameter spaces of the hybrid star EOSs and mass-radius relations are found not sensitive to the low-density hadronic EOS adopted $[16,17]$. 

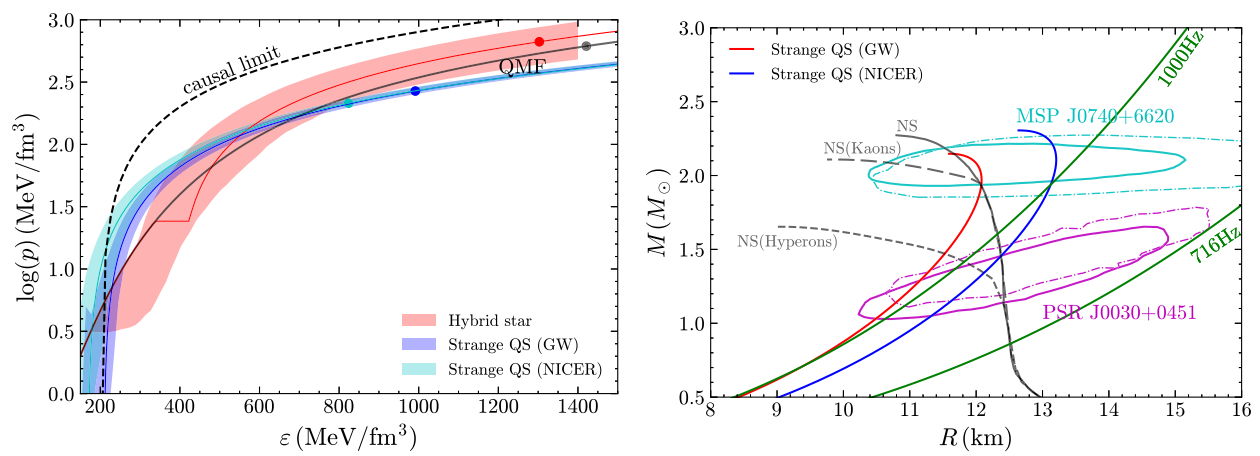

Figure 2. Left: Posterior probability distributions of the EOSs for (hybrid) neutron stars (pink region) $[16,17]$ from combined data of LIGO/Virgo and NICER, in comparison with those of quark stars from LIGO/Virgo (purple region) [15] and from NICER (blue region) [18]. Three dots indicate the densities when the maximum masses of the stars are reached in the most-preferred cases for three analyses (shown in solid lines). The extreme EOS in the causal limit is also shown in the black dashed line. Right: Most preferred mass-radius relations for both (hybrid) neutron stars (black-solid curve) and quark stars (solid red and blue curves) from the EOSs shown in the left panel, along with the microscopic calculations on possible exotic EOSs, like the ones including hyperons (short-dashed curve) [25] and $K^{-}$condensation (dashed curve) [26]. The green lines define the lower boundaries of the mass-radius parameter spaces if certain spin frequencies $(716 \mathrm{~Hz}$ [24], $1000 \mathrm{~Hz})$ are discovered from pulsar-like objects. Also shown are the mass-radius constraints of the NICER mission for PSR J0030+0451 [7, 8] and PSR J0740+6620 [9, 10].

In the left panel of Fig. 2, the posterior distributions of hybrid star EOSs are compared to those of quark stars $[15,18]$, resulting from an analysis based on the widely-used bag-model EOS parametrization incorporating the same data from LIGO/Virgo and NICER. From their comparison, we see that the quark star EOSs become stiff earlier than neutron star ones at low densities and are softer than the neutron star ones at high densities. Consequently, a heavy compact star does not necessarily demand a superconformal sound velocity in the quark star case, and it resulted in a lower (static) maximum mass for quark stars than that of hybrid stars [15]. Note that the maximum mass of neutron star may be lowered due to the appearance of strange baryons (i.e., hyperons, kaons), which competes with that of deconfined quarks in the high-density cores of neutron stars [23].

In the right panel of Fig. 2 the most preferred mass-radius relations for both hybrid stars and quark stars are compared. The dashed and short-dashed curves represent two EOS examples including other types of strangeness phase transitions: The EOS modeling with the inclusion of hyperons [25] are performed in the framework of the Brueckner-Bethe-Goldstone many-body approach with three-body forces and the NSC89 nucleon-hyperon interactions, and the Brueckner theory is combined with a standard chiral model for kaon condensation for the EOS with $K^{-}$[26]. A general comment is that different types of compact stars are not distinguishable from the available data [27]. A possible discovery of sub-millisecond pulsars (represented by the green curve labeling $1000 \mathrm{~Hz}$ ) might not help either, since various theoretical neutron star and quark star models can reconcile with the observation. We expect a more precise radius measurement is possible (at least $\sim 1 \mathrm{~km}$ accuracy) in the near future to change the situation.

In Fig. 3, we include another possible global property possibly to be measured, i.e., the moment of inertia of PSR J0737-3039 A [28], the $1.338 M_{\odot}$ primary component of the first 


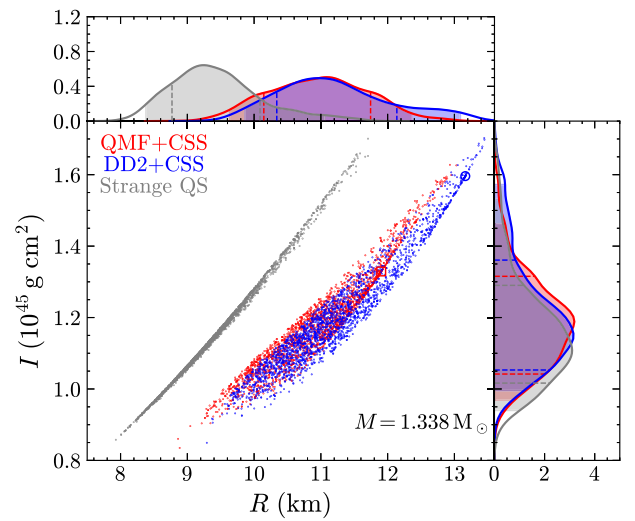

Figure 3. Posterior distributions of $I$ vs. $R$ for a $M=1.338 M_{\odot}$ star, transformed from the EOS parameters posteriors. The probability density of $R$ and $I$ are shown in the upper and right panel, respectively, with the $68 \%$ credible interval in dashed lines and the $95 \%$ credible interval in shade regions. Two markers denote the two results for pure neutron stars. Besides the results of the soft QMF EOS [21], we also include those of the stiff DD2 EOS [29]. Figure taken from [16].

double pulsar system PSR J0737-3039. The moment of inertia of PSR J0737-3039 A is expected to be measured with $\sim 10 \%$ accuracy within the next decade. The posterior distributions of $I$ as functions of $R$ for both hybrid stars and quark stars are shown in Fig. 3. For the analysis of hybrid stars, both the soft QMF EOS [21] and the stiff DD2 EOS [29] are employed for the low-density hadronic EOS. One observes that the two $I$ vs. $R$ distributions are located in separate regions and become more and more separated with each other when increasing $R$, stressing the idea that it can be possible to distinguish them from simultaneous measurement of the stellar radius and the moment of inertia, provided that the accuracy of the radius measurement is at least $\sim 1 \mathrm{~km}$. In particular, we calculate the moment of inertia of PSR J0737-3039 A to be $I_{A}=1.27_{-0.14}^{+0.18} \times 10^{45} \mathrm{~g} \mathrm{~cm}^{2}\left(1.29_{-0.15}^{+0.26} \times 10^{45} \mathrm{~g} \mathrm{~cm}^{2}\right)$ within QMF+CSS (DD2+CSS) as a hybrid star, and a smaller value of $1.13_{-0.13}^{+0.14} \times 10^{45} \mathrm{~g} \mathrm{~cm}^{2}$ as a quark star.

\section{Summary}

The EOS of dense stellar matter is an exciting topic in the area of nuclear astrophysics. Although it is known that the degree of freedom is hadron around nuclear saturation density, the QCD phase state for cold, dense matter for intermediate densities is a very complex problem [30]. A great deal of effort is undergoing in the communities of nuclear physics, particle physics, and astrophysics due to its crucial importance related to the stellar birth and evolution, the merger remnants of neutron star binaries, as well as many puzzles like the central engine of short gamma-ray bursts and the plausible mass gap between neutron stars and black holes. For example, see discussions in our recent review [13].

One key issue of the EOS is still unclear: Does the matter go through a phase transition from hadron matter to quark matter at some intermediate densities, or is quark matter the absolute ground state of strongly interacting matter (i.e., the above-mentioned Witten' conjecture)? After decades of speculation, quark stars still serve as a viable alternative physical model for compact stars. For example, besides the usual binary neutron star merger scenario, binary quark stars have been proposed to be the possible origin for the GW170817 event [31]; Also, it is found that a binary quark star merger for some binary configurations could eject a comparable amount of matter (to the binary neutron star case) [32, 33], to account for the electromagnetic observation in the optical/infrared/UV bands (i.e., kilonova); And a magnetar with quark star EOS is preferred as the post-merger remnant to explain some groups of short gamma-ray burst observations [34, 35].

In the present contribution, we review our recent works on the possibility of distinguishing neutron stars from quark stars from their observed global properties. We mainly find that it is 
possible to distinguish between the two through high-accuracy radius measurement possible in the near future with joint efforts of the multimessenger missions like aLIGO/Virgo, NICER, SKA. Other possible methods include e.g., the post-merger gravitational wave signals with the help of merger simulation, see discussions in e.g., [32, 33, 36-39].

\section{Acknowledgements}

I appreciate discussions with Sophia Han, Jinliang Jiang, Baoyuan Sun, Shaopeng Tang, Chengjun Xia, Enping Zhou, Xia Zhou, Zhenyu Zhu, and the XMU neutron star group. I am also thankful to the latter for assistance with figures. The work is supported by the National SKA Program of China (No. 2020SKA0120300), the National Natural Science Foundation of China (grant No. 11873040), the science research grants from the China Manned Space Project (No. CMS-CSST-2021-B11), and the Youth Innovation Fund of Xiamen (No. 3502Z20206061).

\section{References}

[1] Bodmer A. R., Physical Review D 4, 1601 (1971)

[2] Witten E., Physical Review D 30, 272 (1984)

[3] Arcones A., et al., arXiv e-prints arXiv:1603.02213 (2016)

[4] Antoniadis J., et al., Science 340, 448 (2013)

[5] Linares M., Shahbaz T., Casares J., The Astrophysical Journal 859, 54 (2018)

[6] Cromartie H. T., et al., Nature Astronomy 4, 72 (2020)

[7] Miller M. C., et al., The Astrophysical Journal 887, L24 (2019)

[8] Riley T. E., et al., The Astrophysical Journal 887, L21 (2019)

[9] Miller M. C., et al., The Astrophysical Journal 918, L28 (2021)

[10] Riley T. E., et al., The Astrophysical Journal 918, L27 (2021)

[11] Abbott B. P., et al., Physical Review Letters 121, 161101 (2018)

[12] Xia C., Zhu Z., Zhou X., Li A., Chinese Physics C 45, 055104 (2021)

[13] Li A., et al., Journal of High Energy Astrophysics 28, 19 (2020)

[14] Miao Z., Li A., Zhu Z., Han S., The Astrophysical Journal 904, 103 (2020)

[15] Miao Z., Jiang J.-L., Li A., Chen L.-W., The Astrophysical Journal 917, L22 (2021)

[16] Miao Z., Li A., Dai Z.-G., arXiv e-prints arXiv:2107.07979 (2021)

[17] Li A., Miao Z., Han S., Zhang B., The Astrophysical Journal 913, 27 (2021)

[18] Li A., Miao Z.-Q., Jiang J.-L., Tang S.-P., Xu R.-X., Monthly Notices of the Royal Astronomical Society 506, 5916 (2021)

[19] Weber F., Progress in Particle and Nuclear Physics 54, 193 (2005)

[20] Li A., Zuo W., Peng G. X., Physical Review C 91, 035803 (2015);

[21] Zhu Z.-Y., Zhou E.-P., Li A., The Astrophysical Journal 862, 98 (2018)

[22] Alford M. G., Han S., Prakash M., Physical Review D 88, 083013 (2013)

[23] Li A., Burgio G. F., Lombardo U., Peng G. X., International Journal of Modern Physics E 17, 1635 (2008)

[24] Hessels J. W. T., et al., Science 311, 1901 (2006)

[25] Burgio G. F., Schulze H.-J., Li A., Physical Review C 83, 025804 (2011)

[26] Li A., Zhou X. R., Burgio G. F., Schulze H.-J., Physical Review C 81, 025806 (2010)

[27] Li A., Peng G.-X., Lu J.-F., Research in Astronomy and Astrophysics 11, 482 (2011)

[28] Lyne A. G., et al., Science 303, 1153 (2004) 
[29] Fortin M., et al., Physical Review C 94, 035804 (2016)

[30] Gorda T., Kurkela A., Paatelainen R., Säppi S., Vuorinen A., Physical Review Letters 127, 162003 (2021)

[31] Zhou E.-P., Zhou X., Li A., Physical Review D 97, 083015 (2018)

[32] Bauswein A., et al., Physical Review Letters 103, 011101 (2009)

[33] Zhu Z., Rezzolla L., Physical Review D 104, 083004 (2021)

[34] Li A., et al., Physical Review D 94, 083010 (2016)

[35] Li A., Zhu Z.-Y., Zhou X., The Astrophysical Journal 844, 41 (2017)

[36] Most E. R., Weih L. R., Rezzolla L., Schaffner-Bielich J., Physical Review Letters 120, 261103 (2018)

[37] Montaña G., Tolós L., Hanauske M., Rezzolla L., Physical Review D 99, 103009 (2019)

[38] Chatziioannou K., Han S., Physical Review D 101, 044019 (2020)

[39] Zhou E., Kiuchi K., Shibata M., Tsokaros A., Uryu K., arXiv e-prints arXiv:2111.00958 (2021) 